\title{
Fetal pyelectasis and corkscrew-shaped ureters: an association observed in postmortem fetal imaging studies of osteochondrodysplasia and trisomy 21
}

\author{
'Department of Radiology, University Hospitals Leuven, Belgium \\ ${ }^{2}$ Department of Obstetrics and Gynaecology, University Hospitals Leuven, Belgium \\ ${ }^{3}$ Department of Pathology, University Hospitals Leuven, Belgium \\ ${ }^{4}$ Department of Human Genetics, University Hospitals Leuven, Belgium \\ *Correspondence to: Filip Claus. E-mail: filip.claus@uzleuven.be
}

Kenneth Carels', Luc De Catte ${ }^{2}$, Philippe Moerman ${ }^{3}$, Thomy de Ravel ${ }^{4}$, Raymond Oyen ${ }^{1}$, Jan Deprest ${ }^{2}$ and Filip Claus ${ }^{1}{ }^{*}$

Funding sources: None

Conflict of interest: None declared

Fetal pyelectasis, defined as dilation confined to the renal pelvis, is a common and often transient prenatal sonographic finding. In association with other sonographic markers, a higher incidence of chromosomal abnormalities, in particular trisomy 21, has been reported. ${ }^{1,2}$ Several authors already discussed the clinical significance of fetal pyelectasis, but very few data exist on the underlying structural pathology causing the non-transient pelvic dilation in syndromic cases. ${ }^{3}$

From December 2008 until September 2011, a prospective study was conducted at our hospital to determine the value of high-resolution postmortem magnetic resonance imaging (MRI) as a substitute for or adjunct to conventional autopsy examination following fetal stillbirth or termination of pregnancy (TOP) for medical reasons. A total of 150 pregnancies was included in this study (stillbirth $n=32$, TOP $n=118$ ). The median gestational age at the time of MRI autopsy was 22 weeks (range 12-39 weeks, P25-75 $=19-27$ weeks). Pyelectasis was present in seven cases: five were terminated for osteochondrodysplasia and one for trisomy 21, and there was one case of fetal death after preterm premature rupture of the membranes in a pregnancy with an intrauterine device. The types of osteochondrodysplasia were chondrodysplasia punctata $(n=2)$, thanatophoric dysplasia $(n=2)$, and campomelic dysplasia $(n=1)$. The fetal renal pyelectasia (criteria by Benacerraf et al. ${ }^{2}$ ) was a persistent finding on all prenatal sonographic examinations in the five cases with osteochondrodysplasia and the single case with trisomy 21. In these six syndromic cases, a striking bilateral spiral course of the entire ureter was observed, which was not present in any of the remaining 144 cases who underwent high-resolution postmortem imaging (Figure 1). No histologic examination was performed on fetal ureter specimens. Among the remaining 143 cases without pyelectasis, there was one other trisomy 21 case and two cases of osteochondrodysplasia (achondrogenesis type II and osteogenesis imperfecta), all of them with a normal (straight) course of the ureters.

In their review on the pathogenesis and natural history of fetal renal pelvis dilatation, Ismaili et al. ${ }^{4}$ discussed several theories that account for the visibility of the renal pelvis during pregnancy. Besides physiologic (transient) changes, the best known structural abnormalities include uretero-pelvic junction (UPJ) stenosis (due to strictures or crossing vessels) and vesicoureteral reflux. The latter is often associated with deformed calyces and dilated, tortuous ureters in case of high-grade and chronic reflux. No pyelocalyceal deformation, loss of renal parenchyma, or obvious dilatation of the ureters was seen in the six syndromic cases discussed in this manuscript. This observation pleads against vesicoureteral reflux as the underlying cause of pyelectasis and spiral ureteral twisting in these cases. Also, no crossing vessels were observed, a well-known extrinsic cause of UPJ stenosis.

Segmental spiral twisting of the fetal ureters, sometimes referred to as persistent fetal folds or convolutions, has already been described in the pre-imaging era. ${ }^{5}$ These spiral kinks can occur at any ureteral segment but are most marked at the lumbar third. Few imaging reports exist on the observation of a 'corkscrew' appearance of the ureter in the absence of a lower urinary tract obstruction or documented reflux nephropathy. Kirks et $a l^{6}{ }^{6}$ reviewed the excretory urograms of 100 infants $(\leq 2$ years old) in a retrospective study prompted by a postmortem examination of the urinary excretory system of a newborn who died of a non-renal cause, showing a tortuous appearance of the upper ureteral segments. Apart from intraluminal projections of the ureteral wall, that is, transverse folds, on this postmortem exam, microscopic examination showed a normal wall anatomy. In their review of 100 infants without documented vesicoureteral reflux at cystourethrography, upper ureteral folds were frequently 

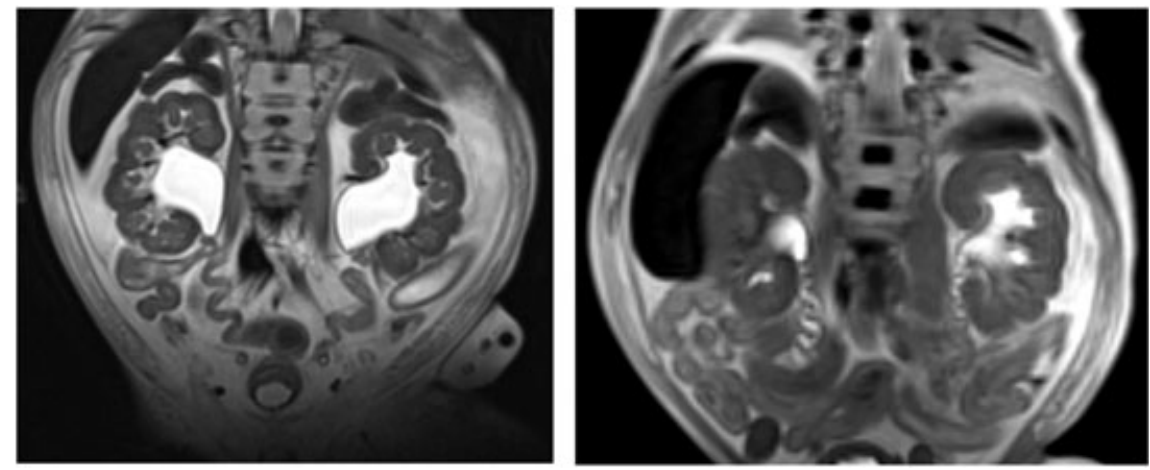

Figure 1 T2-weighted coronal magnetic resonance imaging of the urinary excretory system of two terminated cases showing excessive bilateral twisting of the ureters in the presence of pyelectasis (left case: chondrodysplasia punctata, right case: trisomy 21).

observed in newborns $(75 \%)$ and then gradually decreased with an incidence of $11 \%$ at 2 years of age. In an earlier study of 250 cases, using retrograde contrast injections, cast techniques and histological examination, Östling et $a l^{7}$ concluded that these upper ureteral folds were normal developmental structures, hypothesizing a differential growth rate between the urinary excretory tract and the retroperitoneum and spine. Ureteral folds have limited clinical significance in the great majority of cases but might contribute to the genesis of obstructive hydronephrosis. Also, in the context of osteochondrodysplasia and trisomy 21, it is unlikely that the spiral course of both ureters has major clinical significance, given the paucity of postnatal reports dealing with UPJ stenosis in those syndromes. ${ }^{8}$ Nevertheless, the observed bilateral helical course of the entire ureters is likely to be the cause of pyelectasis. A tentative molecular mechanism for the observed ureteral folding in osteochondrodysplasia and trisomy 21 might the underlying connective tissue disorder. ${ }^{9,10}$

\section{WHAT'S ALREADY KNOWN ABOUT THIS TOPIC?}

- Fetal pyelectasis is a common and often transient prenatal sonographic finding.

- If associated with other sonographic markers, a higher incidence of chromosomal abnormalities is reported. No data exists on the underlying structural pathology causing this non-transient pelvic dilation in syndromic cases.

\section{WHAT DOES THIS STUDY ADD?}

- A strong association between fetal pyelectasis and excessive spiral twisting of the ureters is observed in postmortem imaging studies of osteochondrodysplasia and trisomy 21.

\section{REFERENCES}

1. Coco C, Jeanty P. Isolated fetal pyelectasis and chromosomal abnormalities. Am J Obstet Gynecol 2005;193:732-8.

2. Benacerraf BR, Mandell J, Estroff JA, Harlow BL, Frigoletto FD. Fetal pyelectasis: a possible association with Down syndrome. Obstet Gynecol 1990;76:58-60.

3. Josephson S. Antenatally detected, unilateral dilatation of the renal pelvis: a critical review. 1. Postnatal non-operative treatment 20 years on - is it safe? Scand J Urol Nephrol 2002;36:243-50.

4. Ismaili K, Hall M, Piepsz A, Alexander M, Schulman C, Avni FE. Insights into the pathogenesis and natural history of fetuses with renal pelvis dilatation. Eur Urol 2005;48:207-14.

5. Eisendrath D. Congenital strictures and spiral twists of the ureters. Ann Surg 1917;65:552-9.

6. Kirks DR, Currarino G, Weinberg AG. Transverse folds in the proximal ureter: a normal variant in infants. AJR Am J Roentgenol 1978;130:463-4.

7. Östling K. The genesis of hydronephrosis particularly with regard to the changes at the ureteropelvic junction. Acta Chir Scand 1942;86:1-122.

8. Kupferman JC, Druschel CM, Kupchik GS. Increased prevalence of renal and urinary tract anomalies in children with Down syndrome. Pediatrics 2009;124:615-21.

9. Baitner AC, Maurer SG, Gruen MB, Di Cesare PE. The genetic basis of the osteochondrodysplasias. J Pediatr Orthop 2000;20:594-605.

10. Quarello E, Guimiot F, Moalic JM, Simoneau M, Ville Y, Delezoide AL. Quantitative evaluation of collagen type VI and SOD gene expression in the nuchal skin of human fetuses with trisomy 21. Prenat Diagn 2007;27:926-31. 